\title{
Detection of thermal bridges - aims, possibilities and conditions
}

by A. Wróbel* and T.Kisilewicz**

\author{
*AGH University of Science and Technology, Kraków, Poland
}

${ }^{*}$ Cracow University of Technology, Kraków, Poland

\section{Abstract}

The main aim of the paper is to discuss:

- internal and external conditions that allow to gather reliable thermographic data (required temperature difference, climate conditions, temperature fluctuations),

- inspection methods connected with the structure of the building shell (two- or three-layer shell, location of insulation, interior or exterior survey)

- data range needed for thermal bridge evaluation.

Research work oriented on quantitative evaluation of the cold bridges is based not only on experience and thermal images recorded by the authors but also on computer simulation of the multidimensional heat flow in building shell.

\section{Introduction}

One dimensional heat flow assumption may be introduced when modeling heat transfer only in those parts of the building shell that comply with the following requirements:

- flat surface,

- continuous internal structure,

- adequate dimensions (large enough when compared to the wall thickness).

Those parts of the building elements, that do not comply with the above requirements are usually called thermal or cold bridges and should be modeled by the multidimensional heat flow. It must be clearly stated, that it is not possible to avoid the presence of the thermal bridges in buildings. The proper choice of the building technology and correct design of the structural components may sometimes allow to completely escape or at least minimize the influence on the total heat flow of so called "structural bridges". But it is not possible to avoid the negative influence of the "geometrical bridges", that are connected with basic physical phenomena, which take place in the double or triple corners, wall and floor joints, around the openings etc. Importance of the thermal bridges for building users consists in:

- increased heat losses,

- decreased temperature of the internal surface, that may result in local vapor condensation, mold growth and finally air contamination.

Thermographic inspection allows not only instant location of cold bridges, but also specification of the range and surface temperature evaluation. So easy identification of cold bridges may result in certain overinterpretation, especially when narrow measuring range was used and recorded thermal image shows highly contrasted colors but insignificant temperature differences. Because we are not ready to resign from right angles or perpendicular structural joints in buildings we have to accept the physical phenomena that appear in those places. In the similar way, designers should be aware of the thermal bridging effect when they introduce material discontinuity because of the structural reasons and should protect them with thermal insulation. In general, inequality of the heat flow through building shell is unavoidable effect, that should be possibly minimized, but also correctly interpreted and considered in thermal calculations.

Thermal inspection of the existing buildings is not only aimed at answering the question whether cold bridges exist, but how significant they could be for the total thermal heat losses and if mold growth or surface condensation may appear. This kind of approach is connected with transfer from quantitative to qualitative assessment of the building thermographic images.

For this purpose general conditions of the inspection should be specified, that would assure reliable and sufficient data acquisition. Subsequently, common criteria of the objective thermal image evaluation should be precisely stated.

The main difficulty with field inspection of the building shell in real internal and external conditions, so called "in-situ" examination, is connected with non-stationary boundary conditions. Due to the continuous changes of the environmental temperatures, not only material thermal conductivity but also specific heat and density of the material influence in a significant way heat transfer through building component. Instantaneous temperature distribution, recorded during the quick themographic survey does not fit to the stationary heat flow conditions and is not a good base for reliable calculation of the overall heat transmission coefficient value. Influence of non-stationary conditions on the results of inspection may be to some extent limited when inspection is conducted during the carefully selected period with limited and repeatable air temperature fluctuations. The other conditions connected with successful thermographic inspection, like a lack of solar 
radiation, precipitation and intensive air movement, taking into consideration relative humidity, distance, sky and environmental radiation etc., are well known by now.

Thermal requirements, regarding internal surface temperature to avoid critical surface humidity, included in European standard EN 13788 [1] may also be used in this context. Dimensionless temperature coefficient $f_{\text {Rsi }}$ allows to separate evaluation from actual temperature values. Further information regarding dimensionless temperature coefficient $f_{\text {Rsi }}$ will be given in section 3 .

\section{Thermal bridges in multilayer walls}

\subsection{Thermal bridae in structural laver}

An example of the structural cold bridge is shown below. Thermal image with easy to observe temperature differences of the internal surface of the wall is shown in the left part of Fig. 1. Concrete post and horizontal beam are hidden in the structural layer of the wall made of hollow masonry blocks $29 \mathrm{~cm}$ thick and covered with internal lime plaster. External thermographic view of the wall is shown in the right part of Fig. 1. This wall is insulated with the external layer of $10 \mathrm{~cm}$ Styrofoam and thin plaster.

Irregular contour of the well conducting post and horizontal structural beam may be easily seen in thermogram recorded on the internal side of the wall. But temperature differences, that may be observed on the surface of the wall in this area are not significant, so the problems connected with possible mold growth or vapor condensation does not look serious here. In the external thermal picture of this component the result of the presence of well conducting material is not visible any more. External only thermal inspection of this wall, would not allow to identify this kind of thermal bridge.

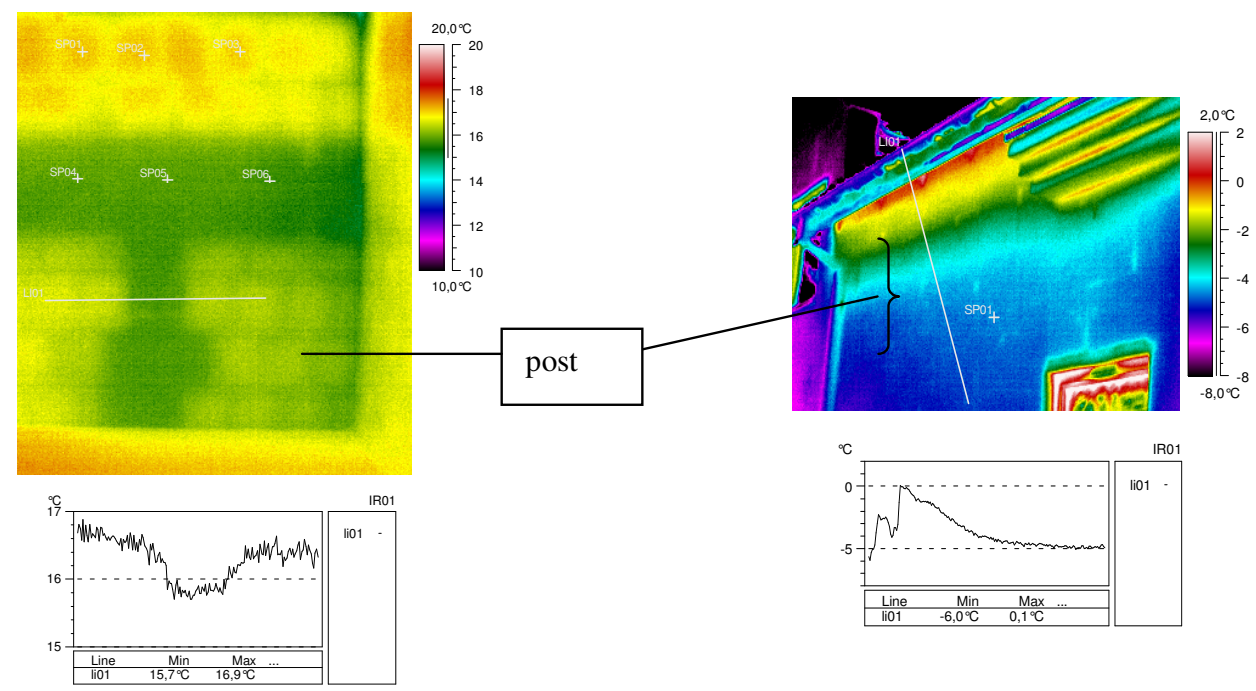

Rys. 1 Irregular concrete post and horizontal structural beam In 2-layer wall, internal air temperature $17.8^{\circ} \mathrm{C}$, external air temperature $-6^{\circ} \mathrm{C}$

Computer simulation of the two dimension stationary heat flow, conducted in program THERM [2], confirms in general way the above observations, Fig. 2. Temperature distribution is practically uniform in external part of styrofoam (parallel isotherms at the bottom of the right part of Fig. 2) and differentiated at internal surface of the wall, as shown in the left part of Fig. 2. There is a discrepancy between the measured and simulated temperature differences, due to unknown material features, irregular structure of the post and most probably due to nonstationary heat flow conditions.

As it was stated above, this kind of the structural inclusion, made of the well heat conducting material should not result in any problems connected with moisture build-up or hygienic conditions. 

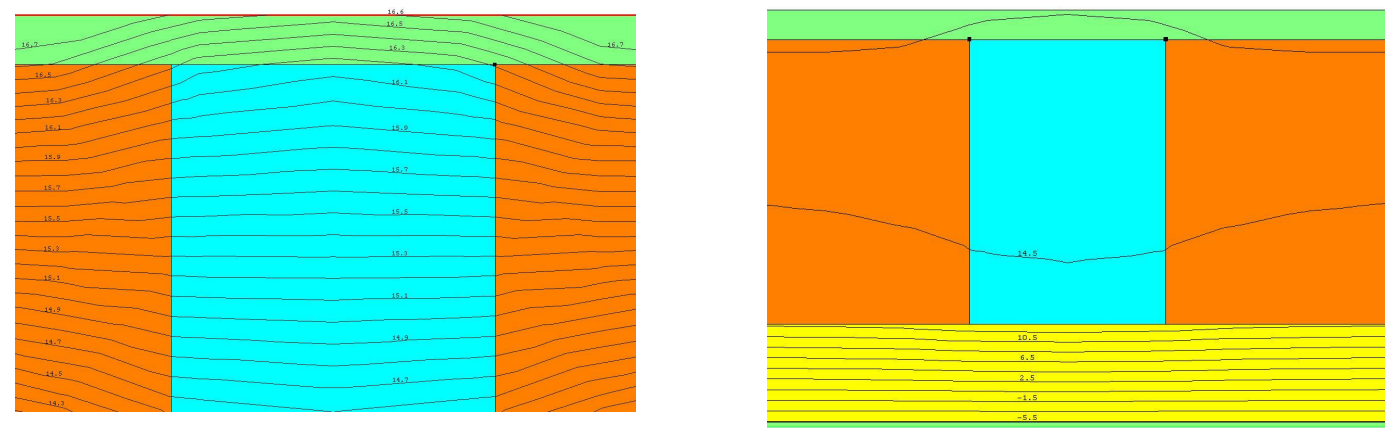

Fig. 2 Temperature distribution in the wall section, 2D heat flow simulation, left side: magnified internal part of the simulated wall with increased isotherm resolution, right side: whole wall section

But if the main aim of the thermographic inspection is taking inventory of the building structure, external inspection of such an object would not bring the expected results and structural elements would not be discovered.

\subsection{Thermal bridge at the meeting point of external wall and roof}

In Polish designing practice, especially regarding the small buildings, usually there is no accurate analysis or detailed sketch of the thermal insulation arrangement in structural joints and the similar building elements. Decision how to solve this problem is usually being left to the contractors and their experience. Incorrect and in the same time a standard way of insulating the meeting point of a 3-layer external wall and pitched insulated roof is shown in Fig. 3. Thick, internal structural layer of the wall goes up to the roof sheathing. There is a lack of insulation continuity between external wall and roof.

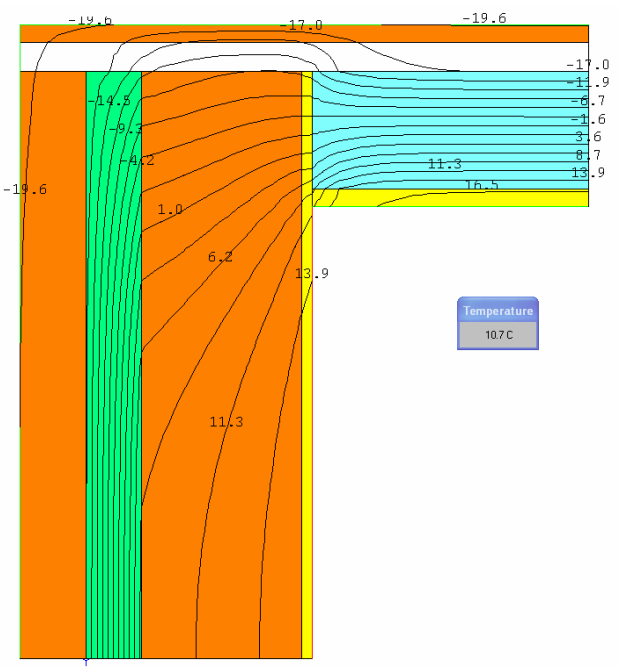

Rys.3 Layer arrangement and isotherm layout at the meeting point of 3-layer external wall and insulated pitched roof, internal air temperature $+17.5^{\circ} \mathrm{C}$, external air temperature $-20^{\circ} \mathrm{C}$.

The lowest internal surface temperature value occurs right at the corner. When external air temperature is $-20{ }^{\circ} \mathrm{C}$ the lowest surface temperature drops down to $11.6{ }^{\circ} \mathrm{C}$. When both environmental temperatures are the same as during the thermal inspection of the building, Fig. 4, temperature difference of the surface at the corner and far away form the thermal bridge is $2.4 \mathrm{~K}$ and fits well to the recorded temperature difference.

As it was shown earlier, it will not be possible to spot this kind of thermal bridge as shown in Fig. 3 and 4 during external only thermal survey of the wall or roof. 


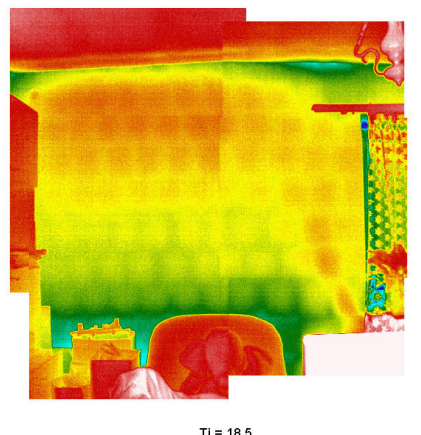

$\mathrm{Ti}=\mathbf{1 8 . 5}$

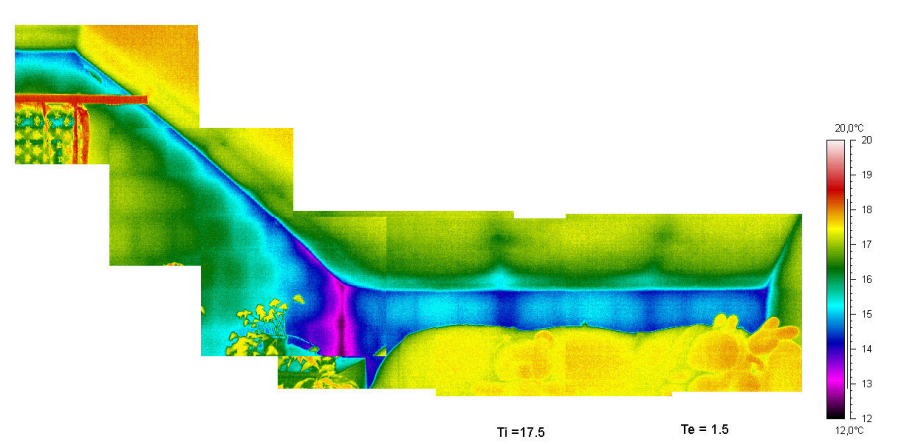

Rys. 4 Thermal image at the meeting point of 3-layer external wall and insulated pitched roof, internal air temperature on the left side is $+18.5^{\circ} \mathrm{C}$ and on the right side is $+17.5^{\circ} \mathrm{C}$, ambient air temperature $1.5^{\circ} \mathrm{C}$.

\section{External wall and floor connection}

Thermal image shown in Fig. 5 was taken at the ground floor of single-family house. 50 years old building with external walls made of ceramic brick was thermally refurbished with $10 \mathrm{~cm}$ external layer of styrofoam. Insulation is finished at the level of the floor and not extended on the foundation wall. Thermal image of this fragment is shown in Fig. 5, internal surface on the left side and external on the right side. The structure of this node results in relatively low temperature at the corner of wall and floor surface. During thermal inspection the lowest surface temperature was $15^{\circ} \mathrm{C}$, while wall surface temperature far away from the corner was 19 to $19.5^{\circ} \mathrm{C}$. The direct reason of thermal inspection of this building was mold growth that appeared above the floor. But the momentary temperature layout at thermal bridge surface in existing conditions did not allow to evaluate explicitly a risk of vapor condensation in the other conditions. To extend analysis range, the authors decided to use again two dimensional, stationary computer simulation of this structural joint.
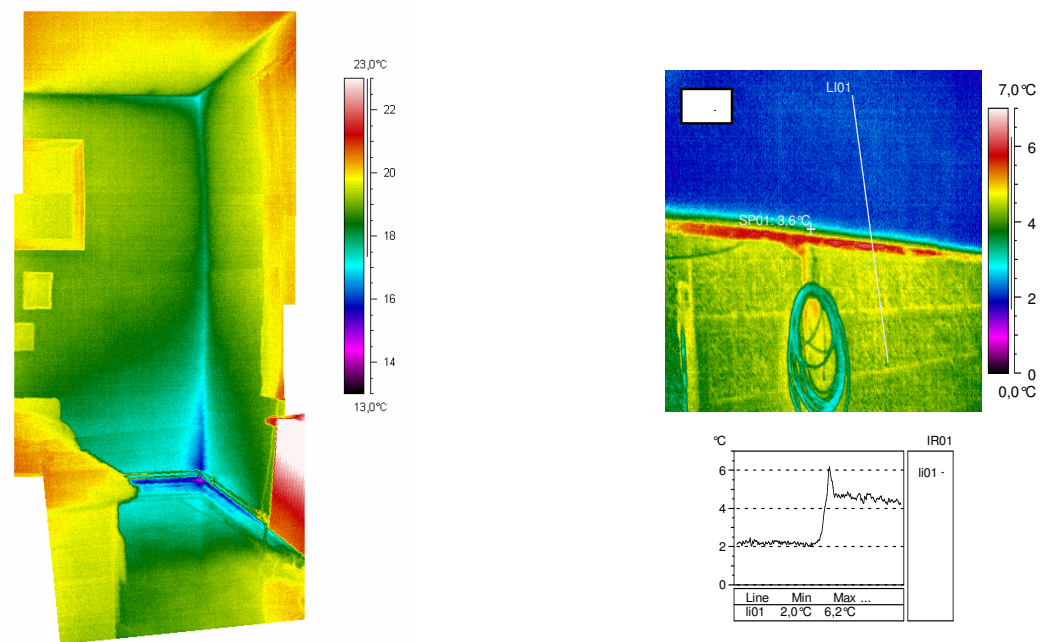

Rys. 5 Structural connection of the external wall, foundation wall and floor upon the ground, internal air temperature $+20.1^{\circ} \mathrm{C}$, ambient air temperature $+3^{\circ} \mathrm{C}$. 




Rys. 6 The computer simulation results, internal air temperature $+20.1^{\circ} \mathrm{C}$, ambientl air temperature $+3{ }^{\circ} \mathrm{C}$.

The results of computer simulation of the analyzed element have been shown in Fig. 6 for the conditions existing during the thermal survey. It was assumed that ground temperature below the floor level is $+8{ }^{\circ} \mathrm{C}$. Surface thermal resistance value of the floor was taken according to European Standard [3] and the same one was taken for adjacent wall to keep the physically continuous model of the connection. The lowest calculated surface temperature, $14.3{ }^{\circ} \mathrm{C}$, was slightly lower than the one achieved during the thermal inspection. Most probably it may be caused by the dynamic external temperature fluctuations in real conditions. In calculations repeated for external temperature equal to $+6{ }^{\circ} \mathrm{C}$ (such a temperature was recorded on the same day 3 hours earlier) surface temperature rises to $+14.9{ }^{\circ} \mathrm{C}$. It may be assumed finally, that the main features of the model of the structural connection are pretty close to the real ones.

This model was used to test the conditions that would occur on the wall surface at the very low, so called computational external temperature, that is equal to $-20.0^{\circ} \mathrm{C}$ in the III-rd climatic zone of Poland. The lowest calculated surface temperature was only $+9.8{ }^{\circ} \mathrm{C}$ (and at $-10{ }^{\circ} \mathrm{C}:+11.8{ }^{\circ} \mathrm{C}$ ). Such a low surface temperature may be the trigger not only for a local mold growth on the wall surface but also for vapor condensation.

Another approach to surface temperature evaluation may be the thermal requirements regarding internal surface temperature to avoid critical surface humidity as in European standard EN 13788 [1] and in a draft of Polish regulations amendment [2], where a concept of dimensionless temperature coefficient $f_{\text {Rsi }}$ (dimensionless temperature) has been introduced.

$$
f_{R s i}=\frac{\Theta_{s i}-\Theta_{e}}{\Theta_{i}-\Theta_{e}}
$$

where:

$\theta_{\mathrm{si}}$ - internal surface temperature,

$\theta_{\mathrm{e}}$ - ambient air temperature,

$\theta_{i}$ - internal air temperature.

Coefficient $f_{R s i}$ may be treated as a kind of a measure of wall insulating quality. For example, surface temperature of the very well insulated wall is close to the internal air temperature. In those conditions dimensionless temperature coefficient $f_{\text {Rsi }}$ value is close to 1.0. Very important aspect of this approach is its independence of the environmental conditions during inspection or simulation. The ratio value of the two temperature differences as in equation (1) depends only on the building shell features but not on the current temperature values of $\theta_{i}$ and $\theta_{\mathrm{e}}$. Thermal resistance of the modern walls with plane surface, parallel layers and one dimensional heat flow is high enough to assure high surface temperature and prevent mold growth even at very low external temperatures. But it is not that easy to meet those expectations in various structural nodes, that become thermal bridges. As it was shown before, surface temperature of the structural nodes may be significantly different than the surface temperature of the plane wall beyond thermal bridge range. 
To prevent mold growth, coefficient $f_{R s i}$ should be bigger than $f_{R s i, m i n}$ value, that corresponds to conditions of capillary condensation in fine pores of building material. Critical $f_{\text {Rsi }}$ value depends on relative air humidity In the building space. In Polish technical requirements amendment draft [2] it is permitted, for average conditions of the living space, to use critical $f_{\text {Rsi,min }}$ value equal to 0.72 .

In case of the thermal bridge that was shown in Fig. 5 , $\mathrm{f}_{\mathrm{Rsi}}$ value is 0.706 . It means that inequality, that prevents mold growth is not satisfied here.

Location and identification of the thermal bridges by means of the thermal imaging inspection, combined with measured environmental temperatures and calculated $f_{\text {Rsi }}$ value allow to evaluate local resistance and a risk of mold growth. Such an evaluation is more general and not limited to the momentary environmental conditions of inspection.

\section{Conclusions}

1. Correctly recorded thermal images of the building external shell may be a good source of not only qualitative but also quantitative data.

2. It is very important to adequately select the conditions of building thermal inspection, i.e. periodical and relatively small air temperature fluctuations and to measure simultaneously both environmental temperatures.

3. In case of multilayer building wall with external insulation or roof with ventilated air space, thermal bridges located in a structural part of the wall, may be identified by internal inspection only.

4. Thermal images of the building may be used for qualitative verification of construction works but also for taking inventory of existing building. In the latter case thermal inspection should be obligatory done on both sides of wall or roof.

5. Computer simulation and calculations, based on thermographic inspection allow to extend evaluation to the other boundary conditions. Such an approach is necessary when comparing results recorded in different environmental conditions.

6. Information obtained by thermographic inspection may be used for more sophisticated quantitative building shell evaluation connected with heat losses, mold growth and surface condensation.

\section{Acknowledgements}

This work was conducted in the frame of the research grant: NN526119133 financed in period 2007-2010 by the Polish Ministry of Science and Higher Education.

\section{REFERENCES}

[1] EN ISO 13788 : 2001 Hygrothermal performance of building components and building elements Internal surface temperatures to avoid critical surface humidity and interstitial condensation - Calculation method.

[2] Technical requirements amendment draft, issued by Polish Minstry of Infrastructure, March 2008.

[3] EN ISO 6946 : 2007 Building components and building element - Thermal resistance and thermal transmittance - Calculation method. 